\title{
LIMITACION ADQUIRIDA EN EL DESEMPEÑO LABORAL Y DESEMPLEO, IMPLEMENTACIÓN DE RECALIFICACIÓN PROFESIONAL EN UN GRUPO DE TRABAJADORES FRUTIHORTÍCOLAS DE LAS PROVINCIAS ARGENTINAS DE RIO NEGRO, NEUQUEN, MENDOZA Y ENTRE RIOS (1997-2010)
}

\author{
ADQUIRED LIMITATION IN WORK PERFORMANCE AND UNEMPLOYMENT. IMPLEMENTATION OF \\ PROFESSIONAL REQUALIFING IN A GROUP OF WORKERS FROM THE ARGENTIN PROVICES OF \\ RIO NEGRO, NEUQUEN, MENDOZA, AMONG OTHERS (1997-2010)
}

\author{
Liliana Sbriller $\mathrm{Z}^{1}$, Ricardo Barrera $\mathrm{D}^{2}$
}

\section{RESUMEN}

El objetivo del presente estudio es mejorar la calidad de la prestación de recalificación profesional para promover la continuidad laboral autónoma en siniestrados con limitación en la actividad (OMS, 2001) que fueron desvinculados de su trabajo a posteriori de un accidente o enfermedad profesional.

Se consideró para el universo el total de los trabajadores despedidos por limitación en el desempeño (39 casos sobre un total de 619 tratados), bajo diferentes circunstancias económicas del país, de la rama fruti hortícola, oleaginosas, industria y afines, pertenecientes a las provincias argentinas de Río Negro, Neuquén, Mendoza y Entre Ríos, entre agosto de 1997 y diciembre de 2010.

El primer paso de la investigación se centró en detectar variables en común a este grupo y definir los aspectos a tener en cuenta para casos posteriores. En segundo lugar se evaluaron tres recursos para promover la continuidad laboral autónoma y los beneficios obtenidos de la prestación de recalificación profesional.

Se utilizó como modalidad de análisis, la investigación basada en la evidencia clínica. Se dividió la muestra en 3 opciones de implementación: 1) Formación profesional 2) Entrega de maquinarias/herramientas y 3) Asesoramiento y entrega de instalaciones para comercios.

A partir de los resultados obtenidos se determinó la opción de "pequeños comercios" como la más beneficiosa, en segundo lugar las "maquinarias y herramientas" y se descartó "formación profesional" para casos de desempleados por limitación adquirida dada la carencia de beneficios encontradas.

Institución: Universidad Nacional de Buenos Aires.

ESTABLECIMIENTO DONDE SE EFECTUÓ LA INVESTIGACIÓN: Productores de Frutas Argentinas Cooperativa de Seguros Ltada.. Anchorena 670. C.A.B.A.

\footnotetext{
${ }^{1}$ Licenciada en Terapia Ocupacional. (ENTO y U.N Mar del Plata). Técnica en Ocra (Universitat Politécnica de Catalunya)- Profesora Adjunta interina Teoría y Técnica de Terapia Ocupacional V . Licenciatura en Terapia Ocupacional. Facultad de Psicología. Universidad Nacional de Buenos Aires. Responsable de área de Recalificación Profesional. Aseguradora de Riesgos del Trabajo Productores de Frutas Argentinas Cooperativa de Seguros Ltda. Terapista Ocupacional de carrera hospitalaria. División de Salud Mental- Hospital General de Agudos Parmenio Piñero. Gobierno de la Ciudad de Buenos Aires. Mail: rosalila01@gmail.com

${ }^{2}$ Técnico Universitario en Estadística de Salud y Registros Médicos.(UBA)
} 
Conclusión: este estudio permitió obtener evidencia clínica del recurso que permitió el mayor beneficio de la prestación para promover la continuidad laboral autónoma de los casos de trabajadores despedidos por limitación adquirida en el desempeño laboral.

\section{Palabras clave:}

Limitación. Desempeño. Desempleo. Recalificación

\section{Abstract}

The aim of the present study is to improve the service of Professional Rehabilitation in order to promote selfsufficient labor continuity for accident victims with activity limitation (OMS, 2001) (1) those who were dismissed from their jobs after an accident or professional disease.

The universe of cases considered was the total of dismissed workers as a result of limited work performance (39 cases out of a total of 619 treated), under the different nationwide economic circumstances, related to fruit and vegetable productions, oilseeds, and linked industries placed in argentine districts of Rio Negro, Neuquén, Mendoza and Entre Ríos, between the months of August 1997 and December 2010.

The first step of the research was centered in detecting common variables in this group and in defining those aspects to take into consideration for future cases. Secondly, three resources to promote self-sufficient labor continuity and the obtained benefits from the use of Professional Rehabilitation were evaluated.

The investigation based on clinical evidence was used as method of analysis. The sample was divided into three options of execution: 1) Professional training 2) Machinery and tools provision 3) Assistance and deliverance of utilities for businesses.

From the obtained results, the option of "small businesses" was determined as priority and in second place "machinery and tools". However, "professional training" was discarded for the cases of unemployed workers due to acquired limitation, because of the lack of benefits found

Conclusion: This study allowed us to obtain clinical evidence of the resource that brought the greatest benefit of the service to promote self-sufficient labour continuity in the cases of dismissed workers as the result of an acquired limitation in work performance

\section{Key words}

Limitation. Performance. Unemployment. Professional. Rehabilitation 


\section{INTRODUCCION}

El objetivo del presente estudio es mejorar la calidad de la prestación de recalificación profesional para promover la continuidad laboral autónoma en siniestrados con limitación en la actividad (OMS, 2001) que fueron desvinculados de su trabajo a posteriori del accidente o enfermedad profesional.

En el marco de la Ley argentina de Riesgos del Trabajo 22457/96 y Resolución 216/03, Recalificación Profesional, las personas siniestradas que presentan desventajas para retornar a su puesto habitual tienen derecho a la Recalificación Profesional, prestación que incluye los diferentes pasos de la rehabilitación profesional (Glarp 2011) como evaluación de la capacidad laboral y análisis ocupacional, determinando, entonces si el trabajador se encuentra en condiciones de retornar a su puesto habitual, en algunos casos con adecuaciones, deberá ser reubicado en otro puesto o requerirá orientación, formación profesional o asesoramiento y organización de un proyecto de micro-emprendimiento y seguimiento, con el objeto de promover la continuidad laboral tras un acuerdo entre empleador y Aseguradora de Riesgos del Trabajo.

Los beneficiarios son trabajadores que tras el accidente o la enfermedad profesional presentan las siguientes desventajas: la tarea agrava su patología de base o secuelas, presenta riesgos para sí o para terceros 0 , finalmente, requieren de una modificación en la modalidad de ejecución

Para Karen Jacobs (1998), el concepto de trabajo está constituido por cuatro elementos: 1) el comportamiento laboral o habilidades pre-vocacionales, 2) las destrezas, la capacidad que el trabajador ha aprendido o tiene la posibilidad de aprender, 3) la aptitud o habilidad temprana como coordinación o inteligencia y 4) las capacidades físicas motoras y senso perceptivas.

Es necesario agregar a este concepto la actitud, es decir, la postura subjetiva del trabajador con respecto a su fuente económica y en este caso la posición que adopta ante la limitación adquirida.

Estos aspectos, que sirven como base para determinar la capacidad laboral actual y futura de un trabajador, resultan insuficientes al momento de justificar un despido. Es necesario acudir, entonces, al concepto de Factores Contextuales Ambientales (Cole Spencer, 2003), Sociales y Culturales, que incluyen aspectos de la situación jurídica, económica, políticas de empleo, situación particular de la empresa empleadora y relación histórica del trabajador en dicha empresa, relación con sus jefes inmediatos y compañeros

Dada la magnitud de variables macro, para este estudio, en particular, se descartaron los casos de despido ajenos a la limitación y debido a factores económicos o por dificultades en la relación laboral previos a la situación actual de demanda. 
Un monto considerable de siniestrados que presentaron desventaja para retornar a su puesto habitual, fueron reubicados en otros puestos o recibieron la adecuación ergonómica del habitual (Bandeo, 2011). Este grupo merecería un análisis posterior.

Para el presente estudio se tomó como universo al total de trabajadores despedidos por limitación en el desempeño (39 casos sobre un total de 619 tratados), bajo diferentes circunstancias económicas de la Argentina, de la rama de fruti-hortícola, oleaginosas, industria y afines, pertenecientes a Río Negro, Neuquén, Mendoza y Entre Ríos, entre agosto de 1997 y diciembre de 2010.

El objetivo de la presente investigación es mejorar la calidad de las opciones de la prestación de recalificación profesional en siniestrados con desventaja profesional que fueron desvinculados laboralmente.

El primer paso de la investigación se centró en detectar variables en común a este grupo y definir los aspectos a tener en cuenta para casos posteriores. En segundo lugar se evaluaron tres recursos para promover la continuidad laboral autónoma y los beneficios obtenidos de la prestación de recalificación profesional.

Todos fueron informados en la primera entrevista sobre los alcances de la prestación y sus derechos en caso de ser desvinculados. Recibieron evaluación de la capacidad laboral y análisis ocupacional del puesto de trabajo, entrevistas familiares, a jefes inmediatos y empleadores con el objeto de construir el perfil ocupacional (American Occupational Therapy Asociation, 2008), (Sbriller, 2012), (Autores varios 2002), comunicación al Empleador acerca de las condiciones de reinserción (Bond, Drake y Becker, 2012), orientación en formación profesional $\mathrm{o}$ asesoramiento en micro-emprendimientos y seguimiento.

Para la evaluación se utilizó la biografía ocupacional (Larson, Wood, Clarck, 2003), el profesiograma de OIT modificado (Schwartz, Enriquez, Fernandez, 2007), en los casos de trastorno músculo esquelético, enfermedad profesional ${ }^{3}$, con evaluación Rula, Test de Michigan o check list Ocra. (Cuenca y Slemenson, C, 2003), Alvarez- Casado, HernandezSoto, Tello-Sandoval, 2009)

A partir de la decisión de la empresa de desvincular al trabajador por no contar con puesto disponible para la reubicación (Ley argentina de Empleo 24.013/91), se inicia la Orientación Laboral, es decir, ayudar a la persona en su elección. El desempeño actual se construye a partir del querer, saber y poder hacer, para relacionarlo con los recursos disponibles en la zona, la situación y tendencia del mercado y las condiciones del puesto, por lo tanto, el nuevo puesto también debe ser evaluado para determinar la factibilidad ( Sbriller y Schwartz, 2003).

\footnotetext{
${ }^{3}$ Los trastornos músculo esqueléticos abarcan diversos problemas a la salud, que pueden dividirse en: lesiones relacionadas con el trabajo de las extremidades superiores conocidas comúnmente como lesiones por microtraumatismos repetitivos (LMR), en las cuales uno de los factores de riesgo es la frecuencia de movimientos, y dolor y lesiones de espalda en las cuales, uno de los factores de riesgo es la frecuencia de manipulación manual de cargas, aunque las extremidades inferiores también pueden verse afectadas pero en menor cuantía. ALVAREZ- CASADO, Enrique y otros. 2009
} 
El proceso de Orientación está dirigido a adultos que se ven sometidos a la elaboración de un doble duelo, la pérdida de parte de su capacidad funcional (discapacidad adquirida) y un despido del empleo, con la consecuente restricción económica y de identificación y pertenencia a un grupo social, que se ve obligado a aceptar la situación, para luego generar un nuevo proyecto de vida (Figley, 2003).

Para la Resolución 216/03, Recalificación Profesional, de la Super Intendencia de Riesgos del Trabajo argentina, esta etapa debe encaminarse hacia la formación profesional con título habilitante en un oficio (de 3 a 12 meses de cursada) o un micro-emprendimiento, en la práctica, la entrega de herramientas o pequeñas maquinarias para ejercer un trabajo autónomo (Serra y Robledo, 2011).

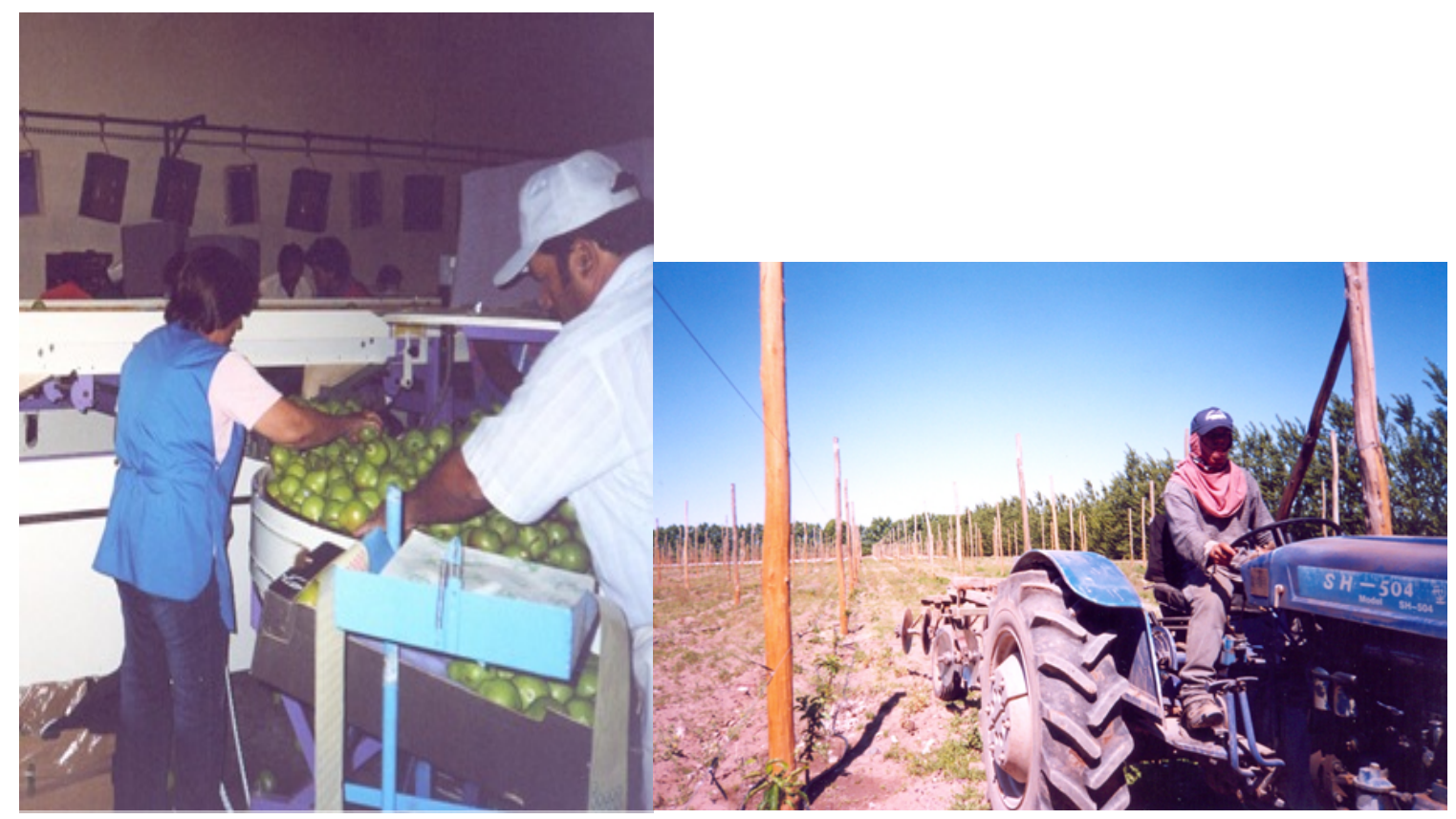

1- CIUO 7414. Operario de la conservación de fruta

2- CIUO 9211. Mozo de labranza

\section{METODO}

El estudio se dividió en dos etapas:

Primera etapa:

a) se determinó el total de casos ingresados al área de Recalificación Profesional (619 casos), se obtuvo la información de los legajos archivados, se respetó la privacidad de datos personales, ya que dada la magnitud temporal de la muestra no fue posible solicitar el consentimiento informado para la investigación. 
b) se seleccionaron los casos que reunían las dos variables: despido y limitación en el desempeño laboral. (39 casos)

c) se evaluaron las siguientes variables:

Factores Personales: sexo, edad, nivel educacional (analfabeto, semi-analfabeto, escolaridad primaria incompleta) o alfabeto (a partir de la escolaridad primaria completa), porcentaje de incapacidad laboral otorgada por comisión médica.

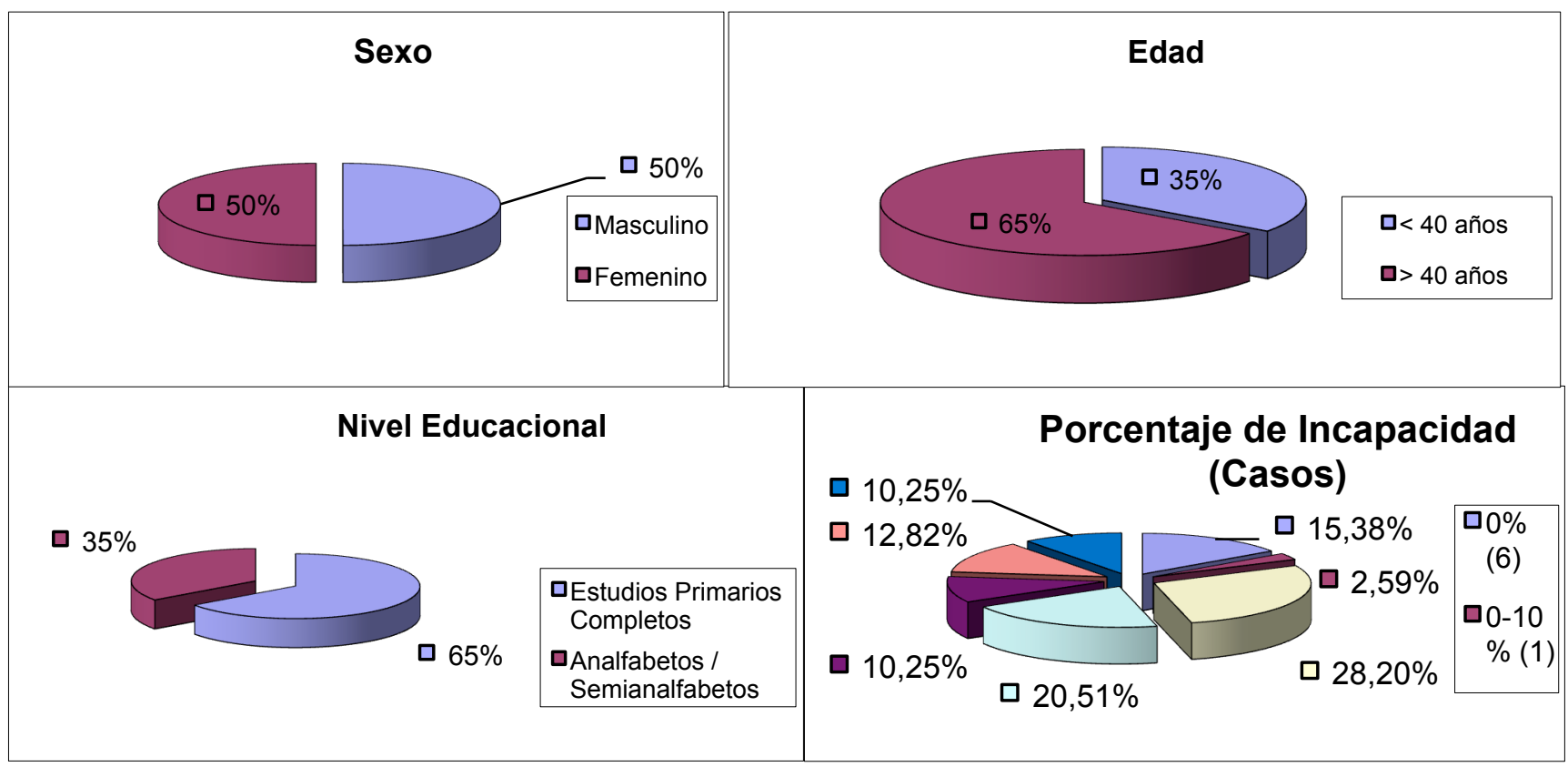

3. Factores personales

Factores Contextuales Ambientales del trabajo: puesto (CIUO, 1988), tipo de siniestro (accidente o enfermedad profesional, los accidentes in itinere se incluyeron en la variable accidente tiempo de permanencia en la empresa de la cual fue despedido, presencia o no de políticas de desvinculación para discapacitados en la empresa,
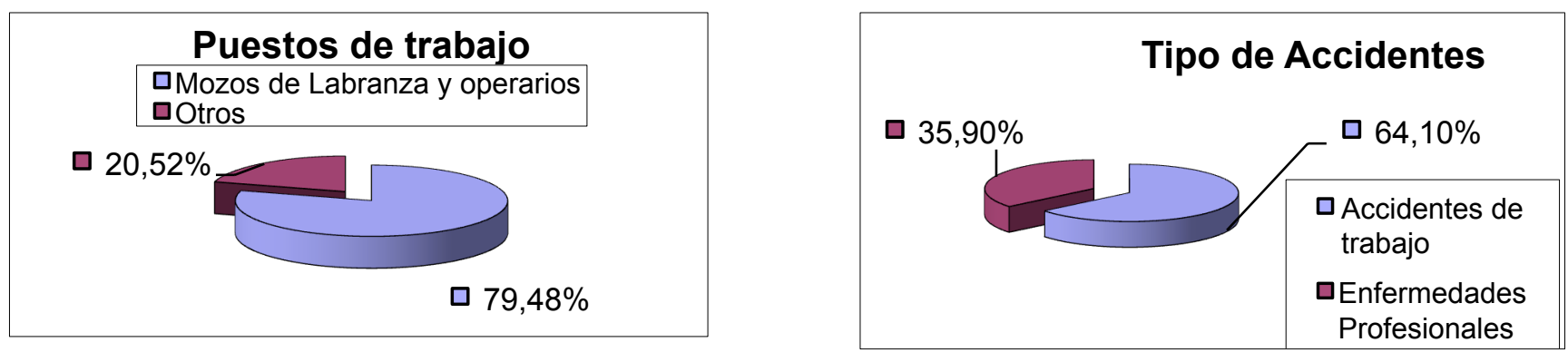

4. Puestos de trabajo- Tipo de accidentes 
Implementación de Recalificación Profesional: tiempo total de la prestación y tiempo transcurrido entre el inicio de la Orientación y la conclusión del caso.

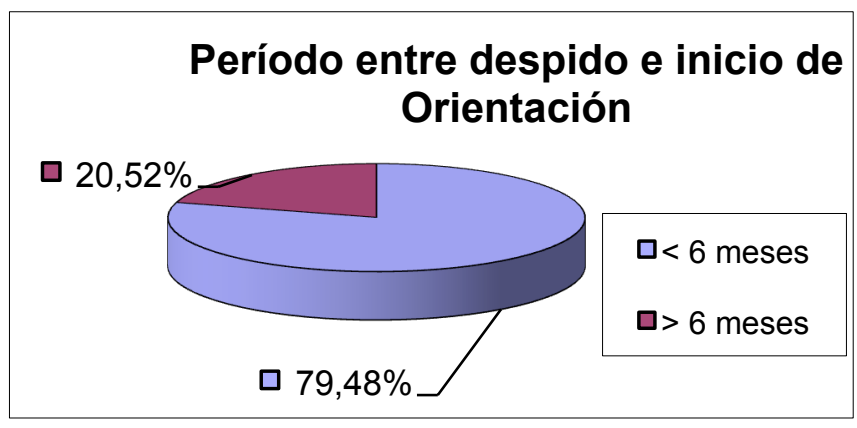

4.Período entre despido e inicio de Orientación

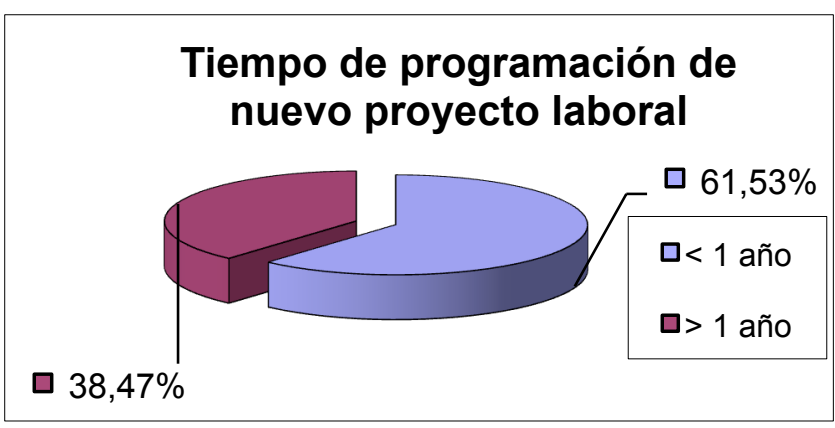

5.Tiempo de programación nuevo proyecto

$4^{\circ}$ Los datos se analizaron de la siguiente manera:

\section{RESULTADOS:}

Factores personales: Edad: la muestra marcó que el $65 \%$ de los casos se concentró en el rango mayor de 40 años, considerado núcleo duro de la fuerza laboral. Sexo: se dividió en partes iguales. Figura 3-

Escolaridad: el $65 \%$ completó sus estudios primarios o más.

El porcentaje de incapacidad otorgado por Comisión Médica analiza fundamentalmente la función perdida a la que se suman pequeños porcentuales (índices de ponderación) donde se considera edad, tipo de tarea y si amerita la prestación de recalificación. No siempre refleja la limitación en la actividad laboral, a sabiendas, situacional y relativa a Factores Contextuales Ambientales que no son tenidos en cuenta para el dictamen.

Esta Comisión considera el $100 \%$ como la capacidad total obrera, en función de la cual se determinarán los porcentuales de incapacidad. Para la presente muestra, el $28 \%$ de los casos se concentró en el intervalo que va del 10 al 20\% de incapacidad parcial permanente, en un rango total que va de $0 \%$ ( 4 casos) hasta el $95 \%$, como se mencionó anteriormente, éste intervalo no aparece como significativo al momento del despido, es decir el nivel de incapacidad determinado no fue fundamental para la decisión empresarial de la desvinculación.

Con respecto a los Factores Contextuales Ambientales. La permanencia en el empleo del cual fueron despedidos se promedió en 12,13 años en un rango total que iba de 1 (se incluyeron 4 casos que trabajaron menos de 1 año) a 38 años.

El tipo de tarea sumó un 79,48 \% en la suma de los códigos 7414 (CIUO, 1998) (operarios de la conservación de fruta), figura 1. CIUO 9211 (mozos de labranza), figura 2.-, dato no 
relevante dado que éste grupo de trabajadores ocupa el mayor porcentaje de los trabajadores asegurados. Con respecto a la postura del empleador, las políticas empresariales explícitas o implícitas que promueven el desempleo de personas con limitación adquirida por cuestiones imputables al trabajo, se concentró en el 59\%. Dato a tener en cuenta, el $64,10 \%$ de los siniestros fueron resultado de un accidente laboral. Figura 4.-

Para concluir se evaluó los tiempos requeridos por los trabajadores para poder elaborar el doble duelo y programar un proyecto laboral autónomo como se mencionó anteriormente. Figura 5.-

El artículo 44 de la Ley de Riesgos del Trabajo considera que el tiempo límite para brindar la prestación abarca 24 meses. En el presente estudio este tiempo total dio como resultado que un $61,53 \%$ requirió menos de un año, teniendo en cuenta que la modalidad del área comienza la evaluación a partir del alta sanatorial y muchos de los casos presentados corresponden al tipo "caso grave".

El período requerido desde la comunicación del despido y por consiguiente inicio de la Orientación tuvo una concentración de 79,48\% de los casos en menos de 6 meses.

\section{Etapa 2:}

Se utilizó como modalidad de análisis la evidencia clínica (Stuart, et al 2002) a partir de la pregunta: Cuál es el efecto de la implementación de Recalificación Profesional en esta muestra? con el objetivo de "mejorar la calidad de las opciones de la prestación"

Se dividió la muestra en 3 opciones de implementación:

Formación profesional: 7 casos

Entrega de maquinarias/herramientas: 23 casos, Figura 6

Asesoramiento y equipamiento de instalaciones para comercios (no contemplado en $\mathrm{R}$ 216/03): 9 casos, Figura 7

Se subdividió las 3 opciones en "beneficios, carencia de beneficios, daños, comentarios". 


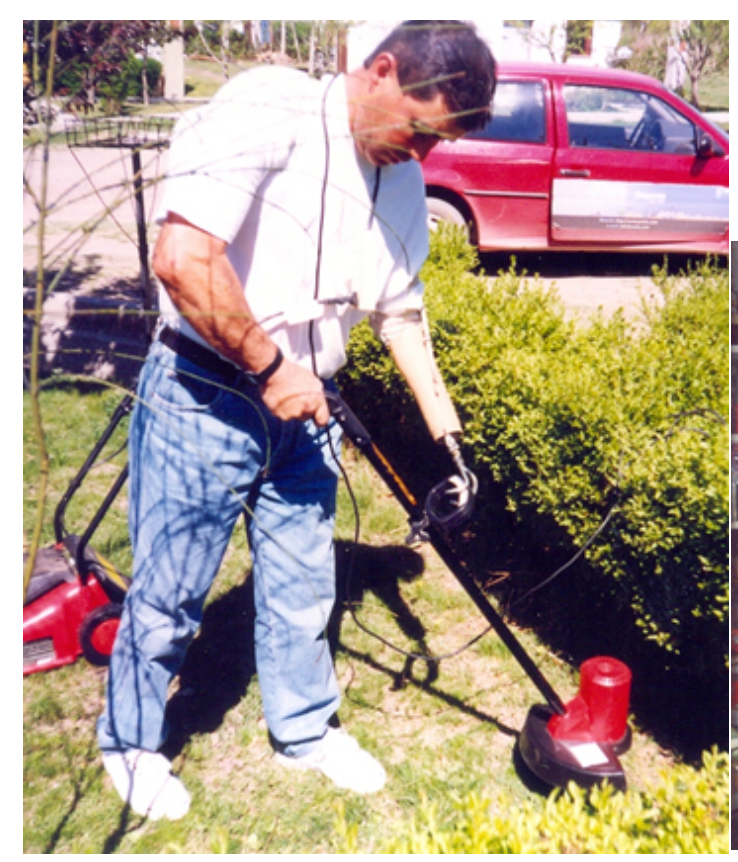

6.Herramientas/maquinarias para trabajo autónomo

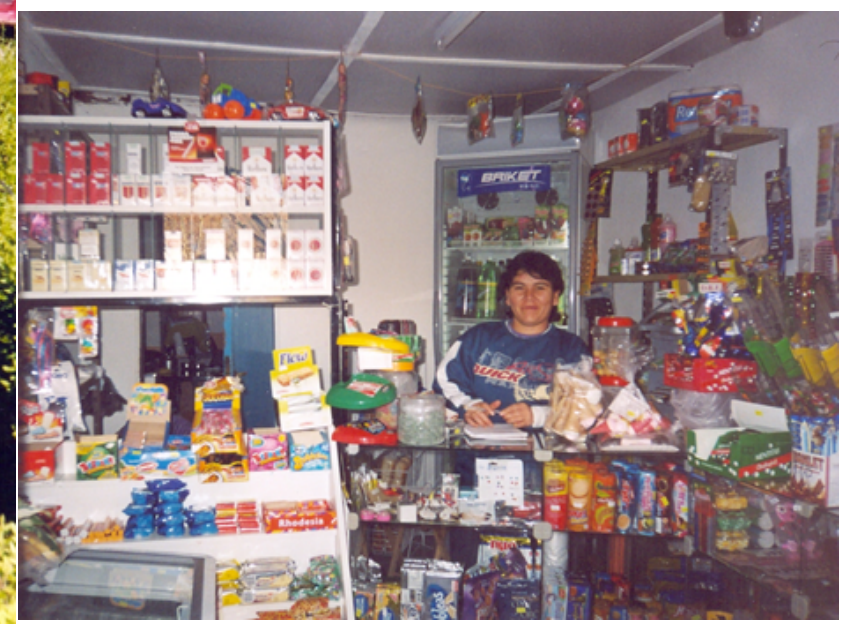

7.Asesoramiento y entrega de instalación de comercios

\section{RESULTADOS Figura 7}

Comentarios preliminares: de los 39 casos analizados, al menos 3 trabajadores (1 de formación y 2 de entrega de maquinarias) volvieron a su puesto habitual en otra empresa, sin tomar en cuenta la indicación de recalificación profesional en casos de desventaja, es decir, no exponerse al riesgo que ocasionó la lesión o enfermedad y sin contralor por parte de las empresas en la evaluación pre-ocupacional.

Evaluados acorde a la modalidad BENEFICIO, CARENCIA o DAÑO, ninguno de los casos obtuvo la denominación DAÑO.

1) Formación Profesional: Dos personas abandonaron el curso por haber conseguido trabajo en otra empresa durante la cursada, con horarios incompatibles. Cinco casos no ejercieron. Del total de 7 casos (100\%), presentaron Beneficio 0\% y Carencia de beneficio 100\%. Por lo tanto ésta opción se considera CARENTE DE BENEFICIOS

2) Micro-emprendimiento: Veintitrés casos recibieron maquinarias o herramientas básicas para oficios que ya ejercían fuera de temporada alta de cosecha fruti-hortícola, por ejemplo, peluquería (set de tijeras, peines y otros), costura domiciliaria (máquina de coser), a veces con objetivos prefijados como la confección de ropa para galgos de carrera en General Roca, Río Negro (actividad específica de la zona), un cavitador para electroestimulación para gabinete estético; maquinarias y herramientas para albañilería, cerrajería, etc. Es decir, continuaron con tareas que tenían como un segundo ingreso económico. Comentarios: 3 de los casos evaluados alquilaron las herramientas a otros trabajadores sin limitación y dos casos retomaron su puesto anterior (contraindicado) en 
otra empresa. Del total de 23 casos (100\%), presentaron Beneficios el 78,26\% y Carencia de beneficios el 21,74\%. La opción se considera BENEFICIOSA.

3) Asesoramiento y equipamiento de instalaciones para comercios: Nueve de las personas que optaron por pequeños comercios, a los que se proveyó de instalaciones básicas desarrollaron su tarea con resultados exitosos, generalmente compartiendo la tarea con familiares (despensas, librerías, tienda de ropa, cibercafé en un barrio humilde en terrenos fiscales y otros). Estos trabajadores contaban con espacio para el emprendimiento y apoyo familiar. Del total de 9 casos (100\%), presentaron Beneficios el $100 \%$ y Carencia de beneficios el $0 \%$. La opción 3 se considera BENEFICIOSA.

\section{Opciones de Implementación}

\begin{tabular}{|l|r|r|r|r|}
\hline & Forración Profesional Maquinarias/Herramientas & Instalaciones para comercios \\
\hline TOTAL & 7 & 23 & 9 \\
\hline Beneficios & 0 & 18 & 9 \\
\hline Carencias & 7 & 5 & 0 \\
\hline
\end{tabular}

7. Tabla de opciones de implementación para orientación

\section{DISCUSIÓN}

El objetivo del presente estudio se centró en mejorar la calidad de la prestación de recalificación profesional para promover la continuidad laboral autónoma en siniestrados con limitación en la actividad (OMS, 2001) que fueron desvinculados de su trabajo a posteriori de un accidente o enfermedad profesional.

La eficacia de la orientación en autoempleo dio como resultado que del total de la muestra de 39 casos, 27 trabajadores $(69,23 \%)$ lograron los objetivos propuestos.

El estudio demostró asimismo la carencia de beneficios de la propuesta de formación profesional, se infiere que fue debido a apremios económicos y limitación en el nivel educacional previo, situación que llevó a algunos de los trabajadores a retomar actividades contraindicadas.

La opción de instalación de pequeño comercio, si bien fue la de mayor éxito mostró dos requisitos inapelables, la situación económica previa, tener un espacio y el apoyo familiar. Muchos de los trabajadores tratados, eran temporarios y oriundos de otras provincias, con un contexto ambiental adverso para la propuesta.

Durante la toma de datos no fue posible tomar como referencia las estadísticas oficiales sobre empleo y desempleo (Indec, 2010) que marcan para el segundo cuatrimestre del corriente año una desocupación del 7,2 \% y subocupación del 9,4\%, ya que las mediciones 
son efectuadas habitualmente en 31 conglomerados urbanos, éste grupo, entonces, queda fuera de la estadística, por lo tanto no permite una medición comparativa en las zonas rurales y pequeñas poblaciones de la argentina para constatar dichos apremios económicos.

Otro hallazgo se presentó en el bajo promedio del porcentaje de incapacidad (centrado entre el 10 y el 20\%) que no permite incluir a los desempleados por accidente o enfermedad profesional en la categorización de discapacidad (Convención sobre los Derechos de las Personas con Discapacidad y Protocolo Facultativo, Naciones Unidas, 2006, si bien todos los casos evaluados presentaban limitación para el desempeño laboral (CIF, 2001) al no quedar incluidos en la citada categoría no percibirían los derechos previstos para casos de discapacidad que según la última encuesta nacional, que no discrimina el origen de la discapacidad (Indec 2004), abarca un 7,1\% de la población.

La Ley de Riesgos del Trabajo ampara en el país a casi 8 millones de trabajadores bajo contrato de trabajo (diario La Nación 30/01/2011) con un índice de siniestralidad de 72,9 empleados por 1000 asegurados en 2009 (cita a publicación de Super Intentencia de Riesgos del Trabajo), prevee la opción de la recalificación como modalidad de orientación del trabajador hacia un autoempleo, pero, pasada ésta etapa deja de acompañarlos cuando pasan a integrar el conjunto de los desempleados, muchas veces en el mercado informal (Bour, Susmel, Roccatagliata, 2010).

La reconversión de una parte de la población de personas con limitación en el desempeño laboral causada por el trabajo incentiva a desarrollar programas de inserción específicos y luego hacer para ellos, esto "se relaciona (Oyarzún, Eugenio, et al, 2010) con las posibilidades de potenciar al Terapeuta Ocupacional como un actor político desde su profesión, político en el sentido de tener perspectivas más globales de la proyección de la disciplina, mantener un manejo de gestión básico para negociar con otros actores, especialmente con aquellos actores del poder político y social que inciden en las políticas públicas de salud vinculadas al desarrollo social del país".

La presente investigación es la primera con casuística sobre el tema en la Argentina, desde 1996 a la fecha se han presentado diferentes cursos y exposiciones sobre la metodología de implementación de la recalificación profesional pero ninguna con estadísticas. Se expone entonces el presente estudio como aporte mediante la investigación basada en la evidencia para el desarrollo de terapia ocupacional en el área laboral

\section{Agradecimientos}

Los autores de ésta investigación agradecen la colaboración desinteresado de los Sres Adrián Elías, Daniel Naón y de la Profesora Marina Tateo. 


\section{REFERENCIAS BIBLIOGRAFICAS}

Alvarez- Casado, Enrique, Hernandez Soto, Aquiles, Tello-Sandoval, Sonia: "Manual de evaluación de riesgos para la prevención de trastornos musculoesqueléticos". ACHS, Edición Especial, Congreso ORP 2009. Editorial FH. Factors Humans.. Primera edición. Barcelona, España, 2009

American Occupational Therapy Asociation: "Marco de trabajo para la práctica de terapia ocupacional: dominio y proceso. Traducción y adaptación al español, Rosa y Ramírez Alicia y cols. Puerto Rico. Publicación y divulgación: www.terapia-ocupacional.com, www.atopronline.org, $2^{\circ}$ edición- 2008.

Autores varios: "Seminario: Formación laboral, empleo y discapacidad, hacia la construcción de criterios comunes". Bs As 2002. Organizadores: Integrándonos para integrar, Puente Verde y Cospe. Apunte de cátedra Teoría y técnica de terapia ocupacional V. Universidad Nacional de Buenos Aires.

Bandeo, Paolo: “Ergonomía: relación vincular entre factores culturales, condiciones de trabajo y la productividad laboral". Trabajo de investigación- . $8^{\circ}$ Congreso Argentino de Terapia Ocupacional, La Plata, Argentina, 2011. Inédito

Bond, Gary, Drake, Robert, Becker, Deborah: "Factibilidad de generalización del modelo de Colocación y Apoyo Individual (IPS) del respaldo al empleo fuera de Estados Unidos. Estudio de investigación. World Psychiatry. Revista oficial de la Asociación Mundial de Psiquiatría (WPA). Edición en español para Latinoamerica. Vol 10, № 2. 2012 www.wpanet.org

Bour, Juan, Susmel, Nuria, Roccatagliata: "Informalidad laboral en la Argentina". Documento de trabajo $\mathrm{N}^{\circ} 102$. Fundación de Investigaciones Económicas Latinoamericanas, mayo 2010 www.fiel.org/publicaciones/Documentos/DOC102

Cole Spencer, Jean: “Evaluación contextual del desempeño”. Unidad 7. Cap. 26. Willard y Spackman: "Terapia Ocupacional" $10^{\circ}$ edición. Recopilación Crepeau- Cohn- Schell Editorial Médica Panamericana. Bs. As 2003- Traducción: Diana Klajn.

Clasificación Internacional Uniforme de Ocupaciones. (CIUO). Listado de Códigos- 1988. www.srt.gov.ar

Convención sobre los Derechos de las Personas con Discapacidad y Protocolo Facultativowww.un/disabilities La Convención sobre los derechos de las personas con discapacidad y su Protocolo Facultativo fueron aprobados el 13 de diciembre de 2006 en la Sede de las Naciones Unidas en Nueva York, y quedaron abiertos a la firma el 30 de marzo de 2007. Se obtuvieron 82 firmas de la Convención y 44 del Protocolo Facultativo, así como una ratificación de la Convención.

Cuenca, Gabriela, y Slemenson, Carlos Resolución Ministerio de trabajo y seguridad social (Argentina) $\mathrm{N}^{\mathrm{a}}$ 295/03. Anexo I (Ergonomía). Manual de Aplicación. Unión de Aseguradoras 
de Riesgos del Trabajo, 2004.-. Apunte de cátedra Teoría y técnica de terapia ocupacional $\mathrm{V}$ (habilitación y rehabilitación profesional). Licenciatura en Terapia Ocupacional. Universidad Nacional de Buenos Aires-

Figley, Charles: “El tratamiento del Stress post-traumático, una perspectiva basada en la teoría". Revista de Psicotrauma para Iberoamérica. Vol II, $\mathrm{N}^{\mathrm{a}}$ 1. Mayo 2003www.psicotrauma.com.ar

Glarp/OIT: “Formación laboral e inserción en el mundo del trabajo". Principios Glarp/OIT, 2011. www.puentesdeluz.org.ar/capacitacion/jornadasdiscapacidad/Glarp_OIT

Honorable Congreso de la Nación Argentina. Ley Nacional de Empleo N ${ }^{\circ}$ 240137/91. Protección del trabajo- 1991-www. infoleg.gov

Instituto Nacional de Estadísticas y Censo. Tasas de empleo y desempleo, segundo semestre 2010. www.indec.gov.ar/empleo

Instituto Nacional de Estadísticas y Censo. Primeros resultados de la encuesta nacional de discapacidad (ENDI), 2004 www.indec.gov.ar/empleo

Jacobs, Karen: "Evaluaciones y programas de trabajo".. cap 8, Areas de ejecución en terapia ocupacional. Unidad II. Evaluaciones y tratamiento en terapia ocupacional. .

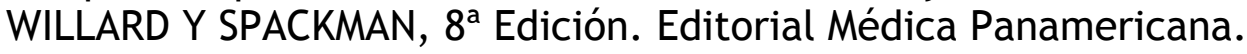

Larson Elizabeth, Wood Wendy, Clarck Florence "Ciencia ocupacional, desarrollo de la ciencia y la práctica de la ocupación a través de una disciplina académica". Willard y Spackman: "Terapia Ocupacional" $10^{\circ}$ edición. Recopilación Crepeau- Cohn- Schell Editorial Médica Panamericana. Bs. As 2003- Traducción: Diana Klajn.

Ley 24557/96 y Resolución 216/2003: Recalificación Profesional. Publicación del Honorable Congreso de la Nación Argentina. Sumario del Boletín Oficial $N^{0} 28242$. Régimen Legal www.srt.gov.ar/normativa/infoleg

Organización Mundial de la Salud-Organización Panamericana de la Salud. "Clasificación internacional del Funcionamiento de la Discapacidad y de la Salud". Edición en español del Instituto de Migraciones y Servicios Sociales (IMSERSO)- Madrid, España- 2001 Traducción: miembros de la Red de Habla Hispana en Discapacidad (RHHD) coordinado por José Luis Vásquez-Barquero. www.conadis.salud.gob.mx

Oyarzún, Eugenio, Acevedo, Carlos: Colectivo Paulo Freire, Olivares. Daniela, Palacios, Mónica, Mendez, Paola , compiladores: Comité Día de la Educación. "Sistematización del día de la Educación". 15 Congreso Internacional. WFOT-03-mayo-2010-Santiago, Chile.

Sbriller, Liliana y Schwartz, Mónica: "Orientación laboral en pacientes psiquiátricos". Trabajo libre IV Congreso Argentino de terapia Ocupacional. Buenos Aires, inédito, 2003.- 
Sbriller, Liliana: “Relación entre educación universitaria y hospitalaria. Implementación del aprendizaje basado en problemas (ABP). Trabajo libre. XXVII ${ }^{\circ}$ Jornadas Multidisciplinaria del Hospital Gral de Agudos P. Piñero- 2010.- Inédito-

Schwartz, Mónica, Enriquez, Celina, Fernandez, Silvina: "Ficha Profesiograma (recopilación y actualización). Inédito. Ficha de Cátedra. Teoría y técnica de TO V. Lic en Terapia Ocupacional- Ftad de Psicología. UBA. 2007.-

Serra, Cecilia, Robledo, Inés: "El rol de terapia ocupacional como parte de la gestión del desarrollo del territorio y el trabajo auto-gestionado". Comunicación libre. $8^{\circ}$ Congreso Argentino de Terapia Ocupacional, La Plata, Argentina, 2011. Inédito

Stang, Silvia: Habrá seguros contra accidentes laborales. Diario La Nación, sección Economía, 30-01-2011

Stuart, Barton y otros, equipo editorial. "Evidencia clínica". La fuente internacional de la mejor evidencia disponible para una atención efectiva en salud". $1^{\circ}$ edición 2002- Basada en Clinical Evidence, edición 6, 2001-Traducción: Centro Cochrane Iberoamericano. Barcelona, España. 